\title{
Immune Dysregulation, Polyendocrinopathy, Enteropathy, X-linked Syndrome in Two Siblings: Same Mutation But Different Clinical Manifestations at Onset
}

\author{
(D) Gülay Karagüzel' ${ }^{1}$, (D) Recep Polat ${ }^{1}$, (D) Mehtap H. Abul2, (D) Alper Han Cebi³, (D) Fazıl Orhan² \\ ${ }^{1}$ Karadeniz Technical University Faculty of Medicine, Department of Pediatric Endocrinology, Trabzon, Turkey \\ ${ }^{2}$ Karadeniz Technical University Faculty of Medicine, Department Pediatric Allergy and Immunology, Trabzon, Turkey \\ ${ }^{3}$ Karadeniz Technical University Faculty of Medicine, Department of Genetics, Trabzon, Turkey
}

\section{What is already known on this topic?}

Though enteropathy, endocrinopathy and skin manifestations are considered the classic triad immune dysregulation, polyendocrinopathy, enteropathy, X-linked (IPEX) syndrome, it is clinically characterized by a wide spectrum of severe autoimmune diseases. The absence of the classic clinical triad of the disease may lead to a delay in diagnosis of IPEX syndrome.

\section{What this study adds?}

IPEX syndrome presented with different clinical pictures in two siblings, despite having the same FOXP3 mutation. IPEX syndrome should be considered in any male infant with only one main disorder typical of IPEX syndrome, such as infantile diabetes, and not only in infants with the full classical triad.

\begin{abstract}
Immune dysregulation, polyendocrinopathy, enteropathy, X-linked (IPEX) syndrome is an early onset systemic autoimmune genetic disorder caused by mutation of the forkhead box protein 3 (FOXP3) gene. Enteropathy, endocrinopathy and skin manifestations are considered the classic triad of IPEX syndrome. However, patients with IPEX syndrome display a variety of phenotypes including life threatening multi-organ autoimmunity. Here, we present the case of two siblings with IPEX syndrome with the same hemizygous mutation, but with different types of symptomology at onset of the disease.
\end{abstract}

Keywords: Immune dysregulation polyendocrinopathy enteropathy X-linked syndrome, neonatal diabetes, renal disease

\section{Introduction}

Immune dysregulation, polyendocrinopathy, enteropathy, X-linked (IPEX) syndrome is an early onset, systemic autoimmune genetic disorder caused by mutation of the forkhead box protein 3 (FOXP3) gene. FOXP3 is located in the short arm of the X chromosome (Xp11.3-q13.3). The FOXP3 gene regulates production and function of the FOXP3 protein which is essential for the regulatory function of $\mathrm{T}$ regulatory $\left(T_{\text {reg }}\right)$ cells $(1,2) . T_{\text {reg }}$ suppress a range of functions of neighboring $\mathrm{T}$ effector $\left(\mathrm{T}_{\text {eff }}\right)$ cells. $\mathrm{T}_{\text {reg }}$ cell dysfunction is the main cause of immune dysfunction in IPEX syndrome, including severe enteropathy, type 1 diabetes, and dermatitis. However, persistent autoreactive B cells and autoreactive $\mathrm{T}_{\mathrm{H}} 17$ cell expansion contribute to autoimmune disorder in this syndrome $(1,2,3)$. Recurrent infections, hemolytic anemia and cytopenia, autoimmune thyroiditis, and other autoimmune disorders, such as hepatitis and nephritis, may be additional manifestations of IPEX syndrome (3).

IPEX syndrome is clinically characterized by a wide spectrum of severe autoimmune diseases, but patients with IPEX syndrome commonly present with early onset, intractable diarrhea within the first weeks of life. If not treated, most 
patients die within the first two years of life because of the consequences of autoimmune manifestations, sepsis, and complications from failure to thrive (2). Nevertheless, there are some reports of patients with longer life. The type and severity of symptoms and the onset of the IPEX syndrome may differ from one patient to another, causing diagnostic delays $(3,4,5,6,7)$.

Here, we present two siblings with IPEX syndrome, with the same hemizygous mutation, but with different types of symptomology at onset; one with dermatitis, type 1 diabetes mellitus and autoimmune hemolytic anemia (AHA) while the other presented with dermatitis, enteritis, and glomerulonephritis. Verbal and written parental informed consents were obtained for both siblings.

\section{Case Reports}

\section{Case 1}

A 5-month old male infant was referred for evaluation for hyperglycemia. His past history and medical records revealed a two-week period of hospitalization at 3-month of age with bronchopneumonia that was treated with antibiotics, and hyperglycemia with normal hemoglobin A1c (HbA1c) and C-peptide levels that was controlled by insulin within three days. According to his family history, this patient was the fourth child of nonconsanguineous, healthy parents and had two healthy elder sisters and one elder brother (case 2) with diagnoses of mesangial proliferative glomerulonephritis, and suspected immune deficiency which was not definitively diagnosed due to the presence of malnutrition, chronic diarrhea, dermatitis, elevated total immunoglobulin E (IgE) levels and recurrent severe respiratory tract infections.

Physical examination at admission revealed a pale infant with eczematous lesions of the neck and scalp. His weight and height were at the fiftieth percentile $(7.3 \mathrm{~kg}$ and 66.5 $\mathrm{cm}$, respectively). The findings from the rest of his physical examination were unremarkable.

His main clinical and laboratory findings at admission are shown in Table 1. Presence of an elevated reticulocyte count (4.49\%), a positive result of a direct Coombs test, and peripheral blood smear showing hemolysis, suggested the diagnosis of AHA. Serum chemical analyses showed an elevated serum glucose with normal blood gas analysis. Urine analysis was normal except for glucosuria. His HbA1c was slightly elevated with reduced and normal fasting and postprandial C-peptide levels $(0.68 \mathrm{ng} / \mathrm{mL}$ and $1.86 \mathrm{ng} / \mathrm{mL}$, respectively, normal range 0.9-7.1 ng/mL). Anti-islet cell and anti-insulin antibodies were normal, whereas anti-glutamic acid decarboxylase was elevated. He was diagnosed as diabetes mellitus and insulin treatment was started.

Having infantile diabetes mellitus, dermatitis and AHA, and an elder brother with suspected immune deficiency, IPEX syndrome was considered. The diagnosis was established genetically by FOXP3 whole gene sequence analysis that revealed a hemizygous p.250K.del (c.748_750delAAG) mutation. His mother was found to be heterozygous for the same mutation. The same mutation has been reported in two previous cases with IPEX syndrome $(7,8)$.

At the age of 1 year the patient underwent a successful hematopoietic, HLA-matched, sibling donor stem cell transplantation (HSCT) with significant improvement in his general course. Dermatitis, and AHA completely resolved at 14 months of age. However, after a 3-month insulin-free period, hyperglycemia recurred and a course of insulin treatment had to be restarted. At the last follow-up at the age of 4.6 years, the patient was in good clinical condition with normal growth and $\mathrm{HbA1 \textrm {C }}$ has been maintained at $<8 \%$ with insulin $(0.8 \mathrm{IU} / \mathrm{kg} /$ day $)$. There are also no accompanying endocrine diseases, with the exception of diabetes mellitus.

\section{Case 2}

This patient is the elder brother of case 1 and the third child of the parents. At the age of 7 months, he was diagnosed with eczematous dermatitis due to skin lesions localized on the neck and trunk. He developed intractable diarrhea and albuminuria when he was around one year old. After that, he was hospitalized several times in different medical centers due to recurrent pneumonia with eczematous skin lesions or albuminuria diagnosed as mesangial proliferative glomerulonephritis by renal biopsy at the age of 18 months. IgE level was as high as $1368 \mathrm{U} / \mathrm{mL}$, while other immunoglobulins were normal (Table 1). Afterwards, he was suspected of having an immune deficiency, as he had malnutrition, chronic diarrhea, dermatitis, elevated total immunoglobulin E levels and recurrent severe respiratory tract infections but this could not be definitively diagnosed. His CD4 and CD8 T lymphocyte counts, NK cells and CD19 B lymphocyte counts were within normal limits. Isohemagglutinins could not be investigated since his blood group was $A B$. He had a positive response againts hepatitis $B$ vaccine but pneumococcal vaccine response was not investigated as the testing was not available. The patient had lifelong problems with mesangial proliferative glomerulonephritis requiring immunosuppression with steroids. After we diagnosed his younger brother (case 1) as IPEX syndrome, the same diagnosis for case 2 was thought probable and was subsequently confirmed by showing the same mutation [hemizygous p.250delK (c.748_750delAAG)] 
in exon 6 of FOXP3 when he was 3.5 years old. The patient died of candida sepsis following severe pneumonia at the age of 4 years. No evidence of diabetes mellitus or other endocrine disease was detected in this patient during his lifetime.

\section{Discussion}

Enteropathy, endocrinopathy and skin manifestations are considered the classic triad of IPEX syndrome $(2,4,5)$. However, patients with IPEX syndrome display a variety of phenotypes, including life threatening multi-organ autoimmunity. We report two siblings with IPEX syndrome, who presented with different clinical manifestations in the infantile period despite having the same mutation in the FOXP3 gene. In addition to dermatitis and immune deficiency findings with recurrent infections, one of the siblings had diabetes mellitus and AHA without enteropathy and the other had mesangial proliferative glomerulonephritis with enteropathy in the first year of the life.

Autoimmune enteropathy is the most common manifestation of IPEX syndrome which present within the early weeks of life with intractable diarrhea. Case 1 had never experienced chronic diarrhea up to last follow up, but case 2 had intractable diarrhea around the age of one.

It has been reported that the frequency of autoimmune endocrinopathies among IPEX patients is $65 \%$, which most commonly include type 1 diabetes and hypothyroidism or hyperthyroidism $(3,5)$. Moreover, it has been suggested that some of the cases of infantile diabetes of unknown etiology might be a mild form of IPEX syndrome (9). Bae et al. (10) reported an IPEX patient who presented with diabetes at 11 months of age but the diagnosis of IPEX was made 10 years later. Therefore, the diagnosis of IPEX should be kept

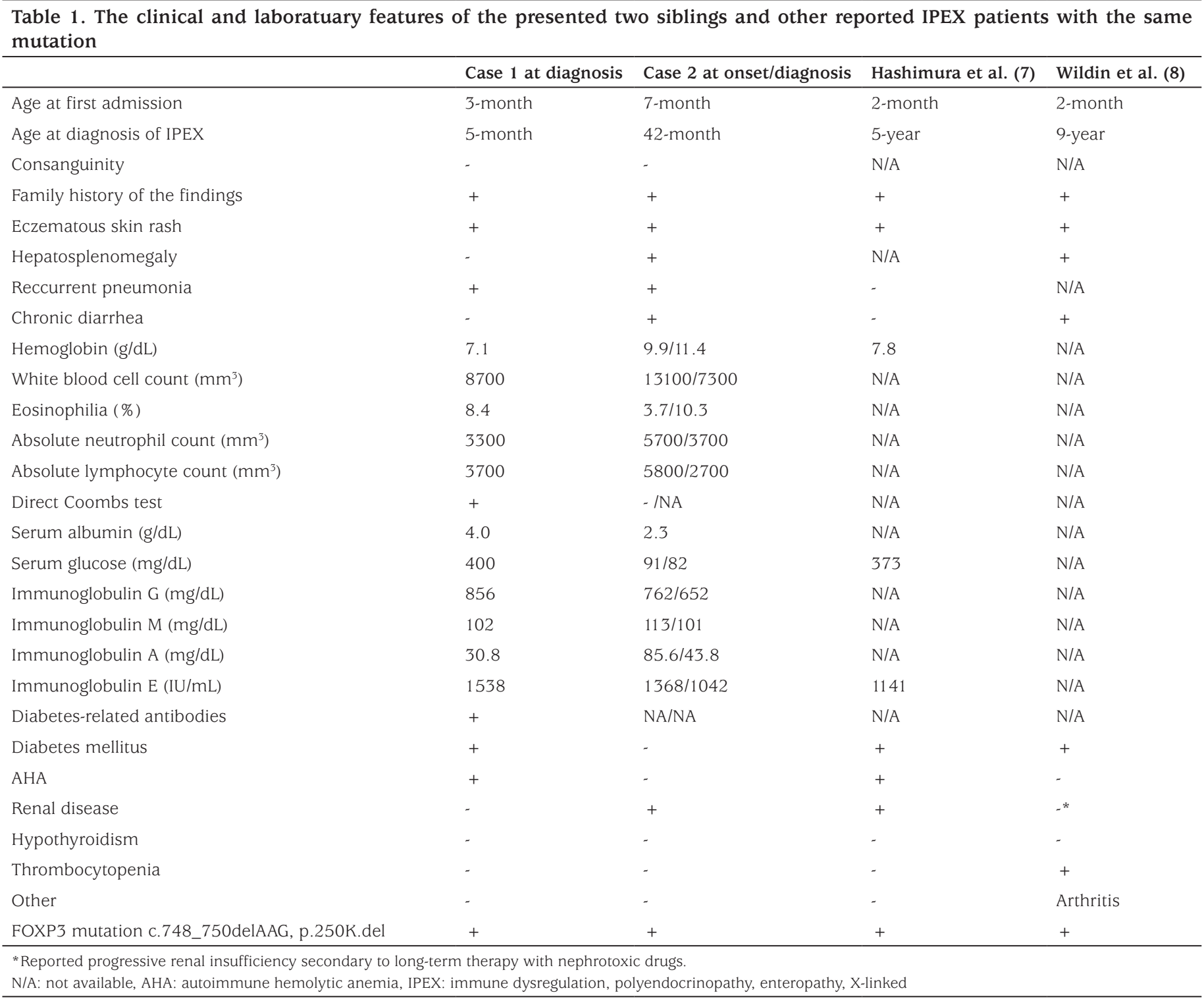


in mind in a male patients who is diagnosed with diabetes in infancy, even though they have no other features of IPEX syndrome initially.

It has been suggested that up to one-third of IPEX patients have renal disease (2). Autoimmunity and long-term usage of nephrotoxic drugs are the main causes of renal disease in these patients. Renal involvement in IPEX syndrome can manifest as tubulointerstitial damage or glomerulopathy presenting as nephrotic syndrome $(2,10,11)$. The mutation detected in the present family has been previously reported in a five-year old boy with IPEX syndrome with minimal change disease (7). Importantly, case 2 presented with a different clinical course from case 1, including mesangial proliferative glomerulonephritis, beside dermatitis, enteropathy, and hyperimmunoglobulin $\mathrm{E}$, and he died at 4 years of age because of severe infection. The genotype and phenotype relationship was evaluated in a large IPEX cohort, but no unequivocal correlation was found (5). The findings in these presented siblings are consistent with a lack of genotype-phenotype correlation in IPEX patients, especially in the first years of the disease. Case 1 had no clinical and laboratory findings of renal disease before and after HSCT. However, Park et al. (11) reviewed the possible genotypephenotype correlation and suggested that enteropathic presentations, eczema, AHA and food allergy were associated with better survival, while thrombocytopenia, septic shock and mutations affecting the repressor domain, intron 7 or poly A sequence were associated with increased risk of death. As the number of reported cases increases, genotype-phenotype correlations may become clearer and its importance in predicting the disease course will become better in the future.

The heterogeneous clinical features of patients with IPEX syndrome can cause difficulties in early diagnosis. The reason for clinical diversity, even in patients with the same mutation in IPEX syndrome, is controversial. Environmental factors have been suggested as a cause of this diversity but we suggest this could not be the case in our patients since they shared similar environmental factors (12). It is of interest whether clinical diversity in IPEX syndrome could be related to the disease onset since the patients were not followed for many years and the actual natural course of the disease and progression is unknown. Furthermore, management of IPEX syndrome may have an important role on the natural course of the disease. That is to say, renal disease and/or enteritis may have developed later in case 1 if the HSCT had not been performed, and case 2 might have developed type 1 diabetes mellitus and/or AHA if he hadn't died. Therefore, we wished to highlight the initial findings in terms of early diagnosis and treatment. The absence of the classic clinical triad of the disease may have led to a delay in diagnosis of IPEX syndrome in our older patient who did not have endocrinopathy. It is also recommended that molecular analysis should be considered to avoid delayed diagnosis.

In the family history of case 1 , the clinical picture of his older brother helped to make an early diagnosis of IPEX syndrome, although case 2 also did not have the classic clinical triad.

\section{Conclusion}

In this context, we would like to underline that family history should be taken in detail and carefully to avoid misdiagnosis regarding IPEX syndrome. We suggest that IPEX should be suspected in a male infant, not only in those with the classical triad, but also in those who have only one main disorder related to IPEX syndrome, such as infantile diabetes.

\section{Ethics}

Informed Consent: Verbal and written parental informed consents were obtained for both siblings.

Peer-review: Externally peer-reviewed.

\section{Authorship Contributions}

Surgical and Medical Practices: Gülay Karagüzel, Recep Polat, Mehtap H. Abul, Alper Han Cebi, Fazıl Orhan, Concept: Gülay Karagüzel, Fazıl Orhan, Design: Gülay Karagüzel, Data Collection or Processing: Gülay Karagüzel, Recep Polat, Mehtap H. Abul, Analysis or Interpretation: Gülay Karagüzel, Alper Han Cebi, Fazıl Orhan, Literature Search: Gülay Karagüzel, Recep Polat, Mehtap H. Abul, Alper Han Cebi, Fazıl Orhan, Writing: Gülay Karagüzel, Fazıl Orhan.

Financial Disclosure: The authors declared that this study received no financial support.

\section{References}

1. Dhuhan BK, Piccirillo CA. The immunological and genetic basis of immune dysregulation, polyendocrinopathy, enteropathy, X-linked syndrome. Curr Opin Allergy Clin Immunol 2015;156:525-532.

2. Bacchetta R, Barzaghi F, Roncarolo MG. From IPEX syndrome to FOXP3 mutation: a lesson on immune dysregulation. Ann NY Acad Sci 2018;141 7:5-22

3. Barzaghi E, Passerini L, Bacchetta R. Immune dysregulation, polyendocrinopathy, enteropathy, X-linked syndrome; a paradigm of immunodeficiency with autoimmunity. Front Immunol 2012;3:1-25.

4. Duclaux-Loras R, Charbit-Henrion F, Neven B, Nowak J, CollardeauFrachon S, Malcus C, Ray PF, Moshous D, Beltrand J, Goulet O, CerfBensussan N, Lachaux A, Rieux-Laucat F, Ruemmele FM. Clinical heterogeneity of immune dysregulation, polyendocrinopathy, 
enteropathy, X-linked syndrome: A French multicenter retrospective study. Clin Transl Gastroenterol 2018;9:201-211.

5. Gambineri E, Mannurita SC, Hagin D, Vignoli M, Anover-Sombke S, DeBoer S, Segundo GRS, Allenspach EJ, Favre C. Ochs HD, Torgerson TR. Clinical, immunological and molecular heterogeneity of 173 patients with the phenotype of immune dysregulation, polyendocrinopathy, enteropathy, X-linked syndrome. Front Immunol 2018;9:2411-2429.

6. Blanco Quiro A, Arranz Sanz E, Bernardo Ordiz D, Garrote Adrados JA. From autoimmune enteropathy to the IPEX (immune dysfunction, polyendocrinopathy, enteropathy, X-linked) syndrome. Allergol Immunopathol (Madr) 2009;37:208-215.

7. Hashimura Y, Nozu K, Kanegane H, Miyawaki T, Hayakawa A, Yoshikawa N, Nakaniski K, Takemoto M, Lijima K, Matsuo M. Minimal change nephrotic syndrome associated with immune dysregulation, polyendocrinopathy, enteropathy, X-linked syndrome. Pediatr Nephrol 2009;24:1181-1186.

8. Wildin RS, Smyk-Pearson S, Filipvich AH. Clinical and molecular feature of the immune dysregulation, polyendocrinopathy, enteropathy, x-linked (IPEX) syndrome. J Med Genet 2002;39:537-545.
9. Hwang JL, Park SY, Ye H, Sanyoura M, Pastore AN, Carmody D, Del Gaudio D, Wilson JF, Hanis JL, Liu X, Atzmon G, Glaser B, Philipson LH, Greeley SAW, T2D-Genes Consortium. FOXP3 mutations causing earlyonset insulin-requering diabetes but without other features of IPEX syndrome: FOXP3 mutations with IPEX-like diabetes. Pediatr Diabetes 2018;19:388-392.

10. Bae KW, Kim BE, Choi J, Lee JH, Park YS, Kim GH, Yoo HW, Seo JJ. A novel mutation and unusual clinical features in patient with immune dysregulation, polyendocrinopathy, enteropathy, x-linked (IPEX) syndrome. Eur J Pediatr 2011;170:1611-1615.

11. Park JH, Lee KH, Jeon B, Ochs HD, Lee JS, Gee HY, Seo S, Geum D, Piccirillo CA, Eisenhut M, van der Vliet HJ, Lee JM, Kronbichler A, Ko Y, Shin JI. Immune dysregulation, polyendocrinopathy, enteropathy, X-linked (IPEX) syndrome: A systematic review. Autoimmun Rev 2020;19:102526.

12. Smith E, Greeley SAW, Ye H, Torgerson TR, Dimmitt R, Atkinson P, Philips J, Goldman F. Exremely early onset IPEX syndrome caused by a novel small exonic deletion in FOXP3. J Pediatr Gastroenterol Nutr 2016;63:119-120. 